Research Article

\title{
Mechanism of Antibacterial Activity of Bacillus amyloliquefaciens C-1 Lipopeptide toward Anaerobic Clostridium difficile
}

\author{
Jia Lv, ${ }^{1}$ Rong Da, ${ }^{2}$ Yue Cheng, ${ }^{1}$ Xiaohong Tuo, ${ }^{1}$ Jie Wei, ${ }^{1}$ Kaichong Jiang, ${ }^{1}$ \\ Adediji Omolade Monisayo, ${ }^{1}$ and Bei Han ${ }^{1}{ }^{1}$ \\ ${ }^{1}$ School of Public Health, Health Science Center, Xi'an Jiaotong University, Xi'an, China \\ ${ }^{2}$ Department of Clinical Laboratory, The First Affiliated Hospital of Xi'an Jiaotong University, Xi'an, China \\ Correspondence should be addressed to Bei Han; hanbei@mail.xjtu.edu.cn
}

Received 11 November 2019; Revised 13 January 2020; Accepted 4 February 2020; Published 4 March 2020

Academic Editor: Frederick D. Quinn

Copyright (C) 2020 Jia Lv et al. This is an open access article distributed under the Creative Commons Attribution License, which permits unrestricted use, distribution, and reproduction in any medium, provided the original work is properly cited.

\begin{abstract}
Probiotics may offer an attractive alternative for standard antibiotic therapy to treat Clostridium difficile infections (CDI). In this study, the antibacterial mechanism in vitro of newly isolated B. amyloliquefaciens $\mathrm{C}-1$ against $C$. difficile was investigated. The lipopeptides surfactin, iturin, and fengycin produced by $\mathrm{C}-1$ strongly inhibited $C$. difficile growth and viability. Systematic research of the bacteriostatic mechanism showed that the C-1 lipopeptides damage the integrity of the C. difficile cell wall and cell membrane. In addition, the lipopeptide binds to $C$. difficile genomic DNA, leading to cell death. Genome resequencing revealed many important antimicrobial compound-encoding clusters, including six nonribosomal peptides (surfactins (srfABCD), iturins (ituABCD), fengycins (fenABCDE), bacillibactin (bmyABC), teichuronic, and bacilysin) and three polyketides (bacillaene (baeEDLMNJRS), difficidin (difABCDEFGHIJ), and macrolactin (mlnABCDEFGHI)). In addition, there were other beneficial genes, such as phospholipase and seven siderophore biosynthesis gene clusters, which may contribute synergistically to the antibacterial activity of $B$. amyloliquefaciens $\mathrm{C}-1$. We suggest that proper application of antimicrobial peptides may be effective in $C$. difficile control.
\end{abstract}

\section{Introduction}

Clostridium difficile is an anaerobic, gram-positive, sporeforming bacterium. Clinical signs of $C$. difficile infection (CDI) range from mild diarrhea to fulminant colitis [1]. The incidence and severity of CDI have increased significantly, especially by the recently emerged and highly virulent epidemic strain BI/NAP1/027 [2]. With increasing antibiotic resistance of $C$. difficile, there is an urgent need to develop new agents and efficient methods for the treatment and control of CDI $[1,3]$. Distinct from the traditional antibiotics, many novel antimicrobial agents, such as ramoplanin, surotomycin, and cadazolid, are currently being investigated in clinical trials for the treatment of CDI. Surotomycin is an orally dosed lipopeptide antibiotic that acts by disrupting the cell membrane [4].
Bacteriocins are ribosomally synthesized antimicrobial peptides with high activity against other bacteria. Bacteriocins are secreted by some probiotic microorganisms, such as Lactobacillus species, Saccharomyces boulardii, and bifidobacteria [5]. Thus, further evaluation should be given to the bacterial resources, antimicrobial mechanisms, and biosafety of probiotics in considering bacteriocins as an alternative or adjunctive therapeutic method for CDI.

Except the ribosomally synthesized antimicrobial peptides, Bacillus species could synthetize a mixture of lipopeptides by nonribosomal peptide synthetases, which mainly include surfactin, iturin, lichenysin, and fengycin families, with broad-spectrum biological activities [6-8]. With the described functional secondary metabolites, many Bacillus spp. strains have been developed as biofertilizers and biopesticides, and they are currently regarded as promising 
environmentally friendly means for plant protection and plant growth promotion, and as secondary metabolite factories [9]. However, for B. amyloliquefaciens, there are only a few reports that describe an antimicrobial activity against toxin-producing C. difficile [10].

We isolated $B$. amyloliquefaciens C-1 from ready-to-eat fruit samples. The bacterial strain is stored in the China Center for Type Culture Collection as a patent strain with the number of CCTCC M2010177. The supernatant of C-1 showed high antioxidant activity and inhibitory activity against foodborne pathogens (Escherichia coli O157:H7, B. cereus, S. aureus, etc.) and human pathogens (C. difficile, Klebsiella pneumoniae, Enterococcus faecium, etc.) [11, 12]; however, no effect against fungi was found.

Comparative genomic analysis showed evolutionary traits for B. amyloliquefaciens strain adaptation to host habitats [13]. The C-1 strain exhibited a biosurfactant activity phenotype against pathogens. The molecular bases/mechanisms of this pathogen-specific activity were unknown. In this study, we investigated the anti-C. difficile mechanisms of the secreted B. amyloliquefaciens C-1 extracellular lipopeptides. We systematically investigated the effects of C-1 lipopeptide on $C$. difficile cell growth, morphological structure, cell wall and membrane integrity, and genome. Then, we resequenced the entire C-1 genome and identified relevant gene clusters, locations, and potential regulatory sequences, including genes for bacteriocins, ribosomally synthesized antibacterial peptides, phospholipase, siderophores, and genes that provide resistance to toxic compounds.

\section{Material and Methods}

2.1. Bacterial Strains and Culture. B. amyloliquefaciens C-1, a patent strain (Chinese patent no. ZL201410260574.2), was isolated and stored in our lab. It was inoculated into fermentation medium (12.4 g/l tryptone, $20 \mathrm{~g} / \mathrm{l}$ glucose, $5 \mathrm{~g} / \mathrm{l}$ $\mathrm{NaCl}, 1.5 \mathrm{~g} / \mathrm{l} \mathrm{K}_{2} \mathrm{HPO}_{4} \cdot 3 \mathrm{H}_{2} \mathrm{O}, 0.04 \mathrm{~g} / \mathrm{l} \mathrm{MnSO}_{4} \cdot \mathrm{H}_{2} \mathrm{O}, 1.7 \mathrm{~g} / \mathrm{l}$ $\mathrm{FeSO}_{4} \cdot 7 \mathrm{H}_{2} \mathrm{O}$, and $1.2 \mathrm{~g} / \mathrm{MgCl}_{2} \cdot 6 \mathrm{H}_{2} \mathrm{O}, \mathrm{pH} 7.2$ ) and grown with shaking of $200 \mathrm{rpm}$ in flasks for $72 \mathrm{~h}$ at $30^{\circ} \mathrm{C}$. Clostridium difficile ATCC 9689, 700057, and BAA-1870 strains were obtained from the American Type Culture Collection and stored at $-80^{\circ} \mathrm{C}$. C. difficile strains were cultured in sterile Reinforced Clostridium Medium (RCM) and incubated overnight at $35^{\circ} \mathrm{C}$ in an anaerobic chamber (Coy Laboratory Products Inc., Ann Arbor, Michigan) with an atmosphere of $82 \% \mathrm{~N}_{2}, 15 \% \mathrm{CO}_{2}$, and $3 \% \mathrm{H}_{2}$.

2.2. Isolation and Identification of B. amyloliquefaciens $C-1$ Extracellular Lipopeptide. Lipopeptide isolation was performed by acid precipitation according to Zhang et al. [14]. Briefly, the crude cell-free culture was adjusted to $\mathrm{pH} 2.0$ with $6 \mathrm{M} \mathrm{HCl}$ and placed overnight at $4^{\circ} \mathrm{C}$. After centrifugation, the precipitate was extracted twice with methanol. The solution was dried in a vacuum freeze dryer, and the dry residue was dissolved in $50 \mathrm{mM}$ Tris- $\mathrm{HCl}$ ( $\mathrm{pH}$ 7.5) and passed through a $0.22 \mu \mathrm{m}$ filter. The extracted lipopeptide sample was analyzed with a UV-VIS spectrophotometer (UV5, Mettler Toledo).
2.3. Thin-Layer Chromatography. The purified lipopeptide was examined by thin-layer chromatography (TLC) on a silica gel G plate [15]. TLC assay was performed on a silica gel G plate $(10 \times 20 \mathrm{~cm}$, Silica gel 60 , Germany). A chloroformmethanol mixture $(10: 1, v / v)$ was used as the mobile phase. A sample was spotted onto the TLC plate and hydrolyzed with $6 \mathrm{M} \mathrm{HCl}$ for $2 \mathrm{~h}$ in a sealed container. Once dried, the plate was developed in the mobile phase. After development, the plate was sprayed with $0.5 \%$ ninhydrin and placed in an oven at $110^{\circ} \mathrm{C}$ for $10 \mathrm{~min}$ to detect the peptides as red spots.

2.4. Semipreparative HPLC Analysis. The putative lipopeptides were identified by HPLC analysis. Briefly, crude extract spots were removed from the TLC plates and dissolved in $10 \%$ methanol; the supernatants were analyzed by semipreparative high-pressure liquid chromatography using an Agilent LC 1200 system. The chromatographic separation was performed with a C-18 Column $(4.6 \times 250 \mathrm{~mm})$. The column outlet was coupled to an Agilent MSD Ion Trap XCT mass spectrometer equipped with an ESI ion source. The lipopeptide fragments were selectively desorbed with methanol gradients from $35 \%$ to $65 \%$ within $140 \mathrm{~min}$. All elution programs used a flow rate of $0.5 \mathrm{ml} / \mathrm{min}$ at $214 \mathrm{~nm}$ and detection occurred using the negative ion mode at $\mathrm{m} / z$ ranging from 400 to 2000 . The isolated fragments were collected for the following experiments.

2.5. Detection of Lipopeptide Synthesis-Related Genes. Lipopeptide biosynthesis genes $(s f r$, ituD, and $f e n B)$ were identified by PCR ( $s f r-\mathrm{F}: 5^{\prime}$ ATGAAGATTTACGGAATTTA $3^{\prime}$, sfr-R: $5^{\prime}$ TTATAAAAGCTCTTCGTACG 3'; ituD-F: $5^{\prime}$ ATGAACAATCTTGCCTTTTTA $3^{\prime}$, ituD-R: 5' TTATTT TAAAATCCGCAATT $3^{\prime}$; fenB-F: $5^{\prime}$ CTATAGTTTGT TGACGGCTC 3', fenB-R: 5' CAGCACTGGTTCTTGT CGCA $3^{\prime}$ ) [9]. PCR conditions consisted of an initial denaturation step at $94^{\circ} \mathrm{C}$ for $5 \mathrm{~min}$ followed by 30 cycles of denaturation at $94^{\circ} \mathrm{C}$ for $1 \mathrm{~min}, 54^{\circ} \mathrm{C}$ annealing for $45 \mathrm{sec}$ $\left(s f r\right.$, ituD) or $1 \mathrm{~min}(f e n B)$, and $72^{\circ} \mathrm{C}$ extension for $1 \mathrm{~min}$ followed by a final extension step at $72^{\circ} \mathrm{C}$ for $7 \mathrm{~min}$. The amplified PCR product was purified and sequenced by an automated sequencer (3730 DNA Analyzer). PCR product sequences were identified using GenBank nucleotide data and BLAST from the National Center for Biotechnology Information, Bethesda, MD, USA (http://www.ncbi.nlm .nih.gov/blast/).

2.6. The Inhibitory Activity of Lipopeptide against C. difficile. Antimicrobial activities of the lipopeptides were detected by disc diffusion assay. $0.5 \mathrm{McF}\left(10^{6} \mathrm{CFU} / \mathrm{ml}\right)$ of $C$. difficile cells was inoculated onto the surface of blood agar plates. An Oxford cup (6 mm diameter), containing $100 \mu$ lipopeptide with concentrations of 5,10 , and $15 \mu \mathrm{g} / \mathrm{ml}$ dissolved in $10 \%$ methanol, was placed on test $C$. difficile-seeded plates. A cup containing $100 \mu \mathrm{l} 10 \%$ methanol was used as negative control. Each $C$. difficile strain was plated in triplicate. The plates were incubated anaerobically overnight at $35^{\circ} \mathrm{C}$, and antimicrobial activity was evaluated by measuring inhibition zones against the tested $C$. difficile cells. The minimal inhibitory concentrations (MIC) against $C$. difficile strains were 
determined by the broth microdilution method in 96-well microplates with a final concentration of $10^{5} \mathrm{CFU} / \mathrm{ml}$, and the final concentration of the added lipopeptide ranged from $10 \mu \mathrm{g} / \mathrm{ml}$ to $0.0095 \mu \mathrm{g} / \mathrm{ml}$; bacterial growth without lipopeptide was set as control [16]. The MIC was defined as the lowest lipopeptide concentration at which growth was completely inhibited after overnight anaerobic incubation of the plates at $35^{\circ} \mathrm{C}$.

2.7. Growth of C. difficile Incubated with Lipopeptides. For time-kill analyses, $0.5 \mathrm{McF}\left(10^{6} \mathrm{CFU} / \mathrm{ml}\right)$ of $C$. difficile cells (strains ATCC 9689, ATCC 700057, and ATCC BAA-1870) was prepared and inoculated into fresh RCM containing 0 , $0.25,0.5$, and 0.75 MIC of lipopeptide separately, and incubated in the anaerobic chamber at $35^{\circ} \mathrm{C}$. Cell viability was determined every $2 \mathrm{~h}$ for $24 \mathrm{~h}$ [17]. Each treatment was performed with three biological replicates.

2.8. Scanning Electron Microscope Analysis of C. difficile Cells Treated with Lipopeptides. An overnight culture of $C$. difficile was transferred into fresh RCM medium with $0.5 \mathrm{McF}$ and $0.25 \mathrm{MIC}$ of lipopeptide, and incubated anaerobically at $35^{\circ} \mathrm{C}$ for $1 \mathrm{~h}$. The $C$. difficile cells were collected and washed three times with sterile PBS solution. The cells were fixed with $2 \%$ glutaraldehyde overnight at $4{ }^{\circ} \mathrm{C}$ and then dehydrated by a graded series of ethanol $(50 \%, 70 \%, 80 \%, 90 \%$, $95 \%$, and $100 \%$ ) for $20 \mathrm{~min}$ at each step. After the critical point of drying and gold coating, the surface structure of treated $C$. difficile cells was observed with a scanning electron microscope (Hitachi S-2460N, Hitachi, Ltd., Tokyo, Japan) at an acceleration voltage of $20 \mathrm{kV}$ [18].

2.9. Fluorescence Microscope Analysis of C. difficile Cells Treated with Lipopeptides. Propidium iodide (PI) penetrates only damaged cell membranes, whereupon it binds to double-stranded DNA and fluoresces red with $488 \mathrm{~nm}$ illumination. To clearly detect an effect on the plasma membrane, 0.5 MIC of the purified lipopeptide was incubated with C. difficile cells $\left(10^{7}\right.$ cells $\left./ \mathrm{ml}\right)$ in RCM liquid medium. The mixture was incubated anaerobically at $35^{\circ} \mathrm{C}$ for $1 \mathrm{~h}$. Then, $10 \mu \mathrm{l}$ of $100 \mu \mathrm{g} / \mathrm{ml}$ PI solution was added to the cell suspension, and the mixture was incubated for $30 \mathrm{~min}$ in the dark. Finally, the treated $C$. difficile cells were observed with a Nikon TI-S fluorescence microscope with the filters set at an excitation wavelength of $488 \mathrm{~nm}$ and an emission wavelength of $538 \mathrm{~nm}$. Cells treated with the same amount of sterile water were used as a negative control [19]. All experiments were repeated three times.

2.10. Determination of Extracellular Alkaline Phosphatase Activity of C. difficile Cells Treated with Lipopeptide. An overnight culture of $C$. difficile was subcultured into fresh RCM liquid medium. Lipopeptide was added separately into the C. difficile culture at final concentrations of 0.25 and 0.5 MIC. Bacterial supernatant $(0.5 \mathrm{ml})$ was collected every $12 \mathrm{~h}$ for the measurement of extracellular alkaline phosphatase (AKPase) activity using an AKP assay kit (Nanjing Jiancheng Technology Co., Ltd., Nanjing, China) as described in [20]. The AKPase unit was defined as $1 \mathrm{mg}$ of phenol produced by $100 \mathrm{ml}$ of bacterial culture supernatant reacted with the substrate at $37^{\circ} \mathrm{C}$ for 15 minutes. Cells treated with the same amount of sterile water were used as negative control. Each test was performed in three biological replicates.

2.11. Lipopeptide Binding to C. difficile Genomic DNA. Gel retardation experiment assays were performed to identify the DNA binding activity of the lipopeptide as described in [21]. Briefly, $50 \mathrm{ng}$ of $C$. difficile genomic DNA was mixed with $1 \mu \mathrm{l}$ of 1,2 , and $5 \mu \mathrm{g} / \mathrm{ml}$ lipopeptide in $20 \mu \mathrm{l}$ of binding buffer (10 mM Tris-HCl, $1 \mathrm{mM}$ EDTA buffer, $\mathrm{pH}$ 8.0). One $\mu \mathrm{l}$ of sterilized water mixed with $C$. difficile genomic DNA was used as negative control. Mixtures were incubated at $35^{\circ} \mathrm{C}$ for $1 \mathrm{~h}$. All samples were subjected to $1.0 \%$ agarose gel electrophoresis and stained with ethidium bromide.

2.12. Whole Genome Sequencing of B. amyloliquefaciens $C-1$. The genomic DNA of strain C-1 was isolated and purified by a kit (Applied Biosystems ${ }^{\circledR} 4413021$ ) and sequenced on the Pacific Bioscience (PacBio) RS II system at Genefund, Shanghai, China. The genome was assembled with SMRT analysis v.2.3 and RS_HGAP_Assembly.3, and the genome assembly was improved by using the software Pilon. Identification of protein-coding open reading frames (ORFs) and annotation of ORFs were performed by using the NCBI Prokaryotic Genome Annotation Pipeline. Genes were functionally annotated by BLAST search in COG (Gene Ontology Consortium), Nr (NCBI RefSeq), and Pfam Databases [22, 23]. KEGG (Kyoto Encyclopedia of Genes and Genomes) database was used in the analysis of metabolic pathways of lipopeptide-producing Bacillus species. All amino acid sequences derived from the Bacillus genomes were submitted to the KEGG database, and the metabolic functions of these sequences were annotated by KASS. The KO (KEGG Orthology) term and corresponding KEGG pathway for each ORF were automatically generated. Secondary metabolite clusters present in the genome of the B. amyloliquefaciens collection have been evaluated using antiSMASH 5.0 [24].

The 16S rRNA gene sequences of Bacillus species were extracted from genome sequences and aligned using the CLUSTALX [25]. Phylogenetic trees were constructed using the neighbor-joining method implemented in the software package MEGA version 7.0.26 [26]. Evolutionary distances were calculated using Kimura's two-parameter model.

The C-1 genome sequence data were deposited into the Sequence Read Archive (SRA) of NCBI and can be accessed via accession number SRP127533.

2.13. Statistical Analysis. All experimental data are expressed as the average with standard deviation of at least three independent replicates. Statistical analyses were performed using the $t$-test and analysis of variance (ANOVA), JMP pro (SAS Institute Inc., NC, US), STAMP ${ }^{10}$, and SPSS V20.0 (IBM Inc., IL, US). Significant differences were considered at $P<0.05$.

\section{Results and Discussion}

3.1. Production, Purification, and Identification of $C-1$ Lipopeptide. Bacillus spp. produces abundant secondary metabolites, such as proteinase, amylase, bacteriocin, and exopolysaccharide. In this study, we focused on the 
lipopeptide-producing B. amyloliquefaciens strain C-1. The growth profile of $\mathrm{C}-1$ is shown in Figure 1. The maximum growth and cell dry weight were reached at $48 \mathrm{~h}$; the maximum lipopeptide production $(3.49 \pm 0.26 \mathrm{mg} / \mathrm{ml})$ was reached at $72 \mathrm{~h}$. Lipopeptide production was conducted in a fermentation medium. The active compound from the culture supernatant was scanned from 190 to $900 \mathrm{~nm}$, and the maximum absorption occurred at $213 \mathrm{~nm}$, which is the typical absorption wavelength of peptides. Three spots were observed by thin-layer chromatography, and three component peaks were detected by RP-HPLC. Mass spectroscopy showed that the molecular masses of the three components at $m / z$ were $1067 \mathrm{Da}, 1477 \mathrm{Da}$, and $1506 \mathrm{Da}$, which corresponded to surfactin, fengycin, and iurin, respectively. PCR products of $675 \mathrm{bp}, 1400 \mathrm{bp}$, and $482 \mathrm{bp}$ corresponded to Srf, FenB, and ItuD genes, and the PCR fragments were sequenced and showed 99\% identity with surfactin, fengycin, and iturin biosynthesis gene clusters, individually (S1).

\subsection{Antimicrobial Activity of C-1 Lipopeptide against $C$.} difficile. Bacillus spp. lipopeptides have an inhibitory activity against plant pathogenic fungi and pathogenic bacteria and have been developed as biocontrol agents [27]. Although, in our previous report, C-1 did not show any inhibitory activity against fungi, it did have antibacterial activity toward several human pathogens, and this antibacterial activity in the C-1 supernatant was verified to be the contribution of lipopeptide, not exopolysaccharide $[11,12]$.

In plate tests, the C-1 lipopeptide displayed antagonistic activities against three $C$. difficile strains. Inhibition zone diameters ranged between $7.05 \mathrm{~mm}$ and $22.00 \mathrm{~mm}$, and the largest inhibition zone was from $15 \mu \mathrm{g} / \mathrm{ml}$ lipopeptide against strain C. difficile ATCC 9689 (Table 1). The MICs against $C$. difficile strains ATCC 9689, ATCC 700057, and ATCC BAA1870 were $0.75,2.5$, and $2.5 \mu \mathrm{g} / \mathrm{ml}$, separately. Within a certain concentration range $(0.0095 \mu \mathrm{g} / \mathrm{ml}-10 \mu \mathrm{g} / \mathrm{ml})$, the inhibitory effect was positively correlated with the concentration of the C-1 lipopeptide. To analyze the inhibitory effect, we determined the growth curves of the three $C$. difficile strains. At $24 \mathrm{~h}$ of continuous measurement, the maximum $\mathrm{OD}_{600}$ of C. difficile ATCC 9689, ATCC 700057, and ATCC BAA-1870 treated with $1 / 4$ MIC lipopeptide reached 51.57\%, 51.54\%, and $56.12 \%$ of the control. For the $1 / 2$ MIC treatment, growth was reduced to $43.15 \%, 46.39 \%$, and $46.12 \%$ of the control. For the 3/4 MIC treatment, growth was further reduced to $38.95 \%, 40.21 \%$, and $41.84 \%$ of the control. The C. difficile ATCC9689 was significantly the most sensitive to the treatment $(P<0.01)$, and the inhibitory effect was stronger with an increased concentration of the C-1 lipopeptide (Figure 2).

Because C. difficile ATCC9689 was more sensitive to the C-1 lipopeptide, and it was also a $t c d A$ and $t c d B$ positive strain, we assessed the following antibacterial mechanism toward this strain.

3.3. Effect of C-1 Lipopeptide on C. difficile Morphology, Cell Wall Permeability. Scanning electron microscopy showed that the surface of $C$. difficile ATCC 9689 was damaged after treatment with the C-1 lipopeptide. Exudates surrounded the

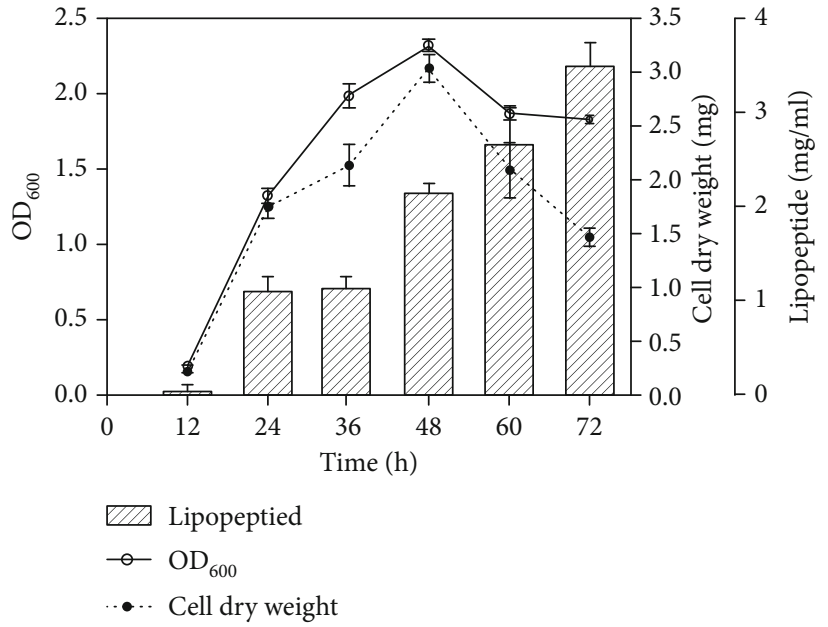

FIgURE 1: The fermentation and lipopeptide production in $B$. amyloliquefaciens C-1 for 72 hours. The solid line indicates the growth curve of $\mathrm{OD}_{600}$, the dotted line indicates the growth curve of cell dry weight, and the shaded columns represent the lipopeptide production.

bacteria, and the cell wall and cell membrane were interrupted and indistinct, whereas untreated cells were smooth and uninterrupted (Figures 3(a) and 3(b)). In addition, with increasing concentrations of lipopeptide, the bacteria were surrounded by exudate that may have been cytoplasm extruded from the cells. Propidium iodide (PI) penetrates only a damaged cell membrane, after which it binds to double-stranded DNA and fluoresces red with illumination at $488 \mathrm{~nm}$. We stained the C-1 lipopeptide-treated and C-1 lipopeptide-untreated $C$. difficile cells with PI and observed the cells with a fluorescence microscope. C. difficile ATCC 9689 cells treated with lipopeptide were stained with PI as shown by red fluorescence, which indicated a damaged cell membrane (Figures 3(d) and 3(f)), whereas the untreated cells did not show any fluorescence (Figures 3(c) and 3(e)).

The destroyed cell membrane increased the permeability of C. difficile ATCC 9689 after C-1 lipopeptide treatment. To verify the effect of lipopeptide on cell wall permeability, we measured alkaline phosphatase (AKPase) activity. A damaged cell wall and cell membrane increased cell permeability and caused an increase in extracellular AKPase. After lipopeptide treatment, extracellular AKPase activity increased continuously and was significantly higher than that of the untreated cells (Fig S2). With $36 \mathrm{~h}$ of incubation, the extracellular AKPase content of C. difficile ATCC 9689 treated with 1/4 MIC and 1/2 MIC of lipopeptide increased 4.7-fold and 7.7-fold.

The antibacterial activity of Bacillus spp. lipopeptide was observed with other pathogens, such as S. aureus [28], Vibrio anguillarum [18], and E. clocae [8]. However, our report is the first to document the effects of lipopeptide on toxinproducing C. difficile. The inhibitory mechanism of the C-1 lipopeptide against $C$. difficile could be explained by destroying the bacterial cell by forming ion-conducting channels in the cell membrane as described by Etchegaray et al. [29]. This mode of action drastically reduces the chance of the 
TABle 1: The inhibition of the C-1 lipopeptide against Clostridium difficile in a plate test (the inhibition diameter showed in mm).

\begin{tabular}{lcccc}
\hline Clostridium difficile & & \multicolumn{3}{c}{ Concentration $(\mu \mathrm{g} / \mathrm{ml})$} \\
\hline ATCC 9689 & 0 & 5 & 10 & $15.50 \pm 0.71^{\mathrm{a}, \mathrm{b}}$ \\
ATCC 700057 & 0.00 & $7.05 \pm 0.71^{\mathrm{a}, \mathrm{b}}$ & $8.40 \pm 0.71^{\mathrm{a}}$ & $22.00 \pm 1.41^{\mathrm{a}, \mathrm{b}}$ \\
ATCC BAA-1870 & 0.00 & 0.00 & $8.50 \pm 0.71^{\mathrm{a}}$ & $11.50 \pm 0.71^{\mathrm{a}}$ \\
\hline
\end{tabular}

${ }^{\mathrm{a}}$ Significant difference of $\mathrm{C}-1$ lipopeptide treatments vs. negative control $(P<0.01)$. ${ }^{\mathrm{b}}$ Significant difference of lipopeptide treatments between $C$. difficile strains ATCC 9689 and ATCC 70057, ATCC 9689, and ATCC BAA-1870 $(P<0.01)$

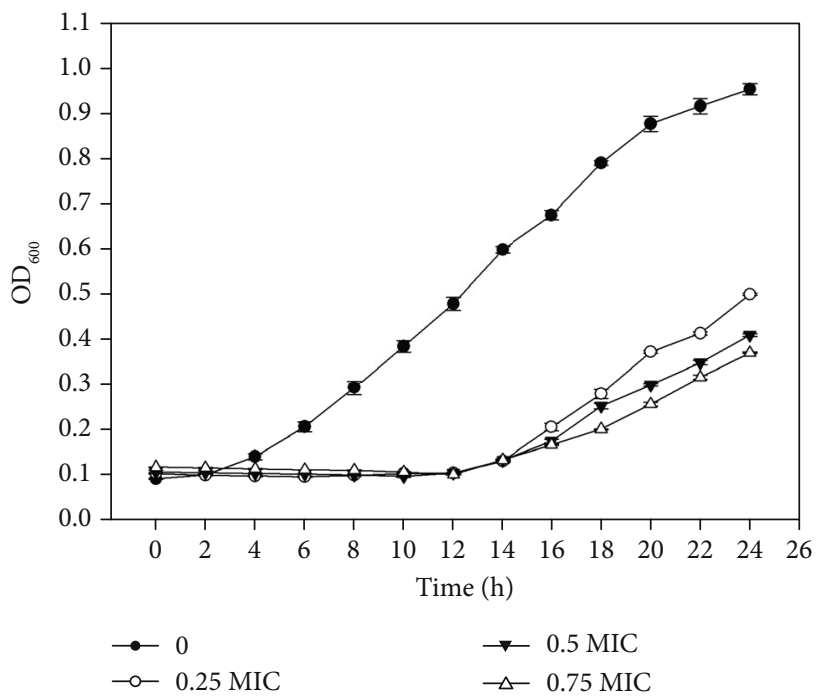

(a)

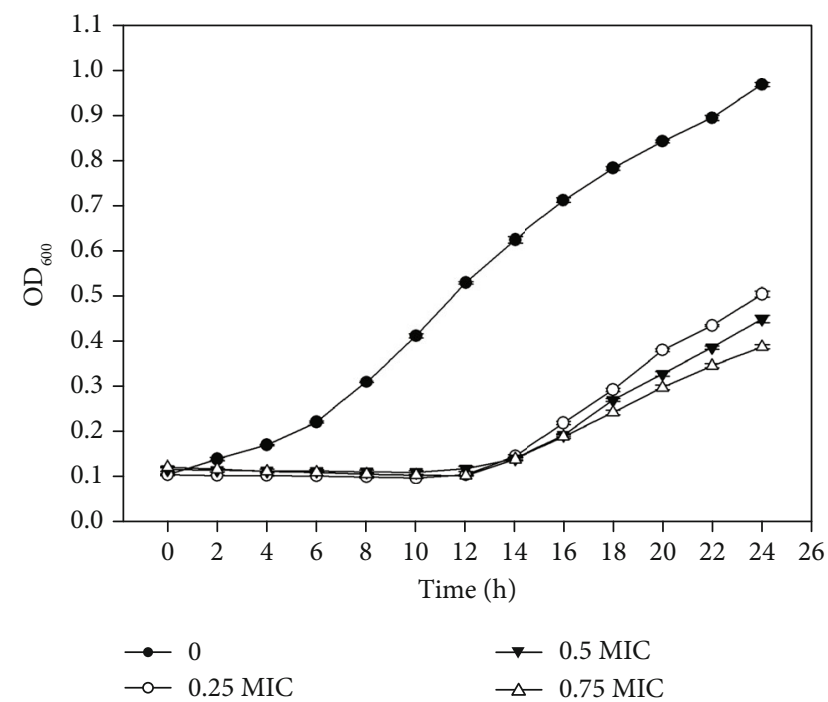

(b)

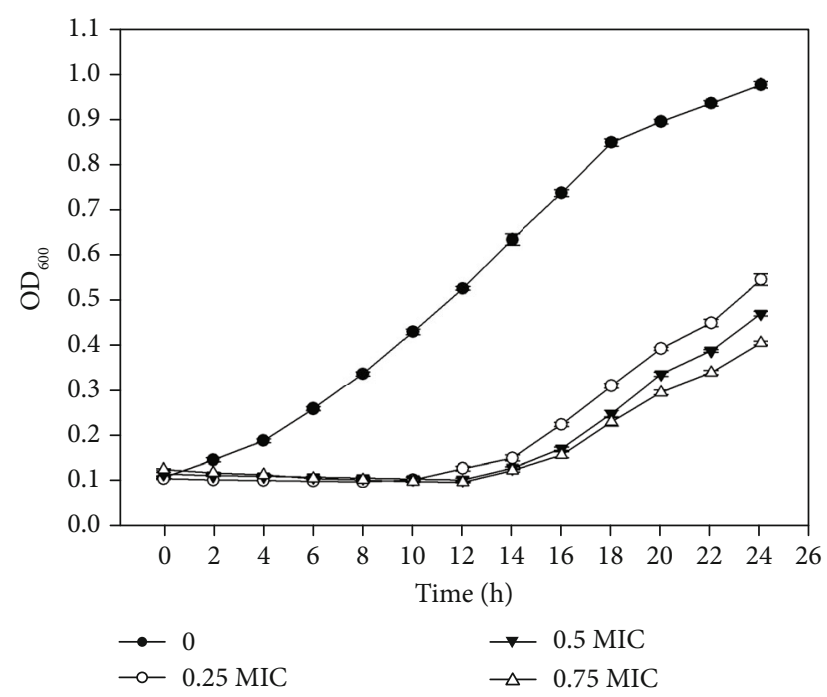

(c)

FIgURE 2: The growth curve of C. difficile ATCC 9689 (a), ATCC 700057 (b), and BAA-1870 (c) treated with different concentrations of the C-1 lipopeptide.

development of resistance by microbes, offering a promising alternative for the treatment of CDI.

3.4. Genome Sequencing of B. amyloliquefaciens $C-1$. The circular chromosome of C-1 contains 3,934,216 bp, 46.5\% GC content, 27 rRNA and 86 tRNA genes (Table 2,
Figure 4(a)). Genome annotation at the RAST server showed that the C-1 genome encodes 4013 proteins, and the corresponding functional categorization by COG annotation is in Figure 4(b). The sequence data of the B. amyloliquefaciens C-1 genome were deposited into NCBI and can be accessed via accession number SRP127533. 


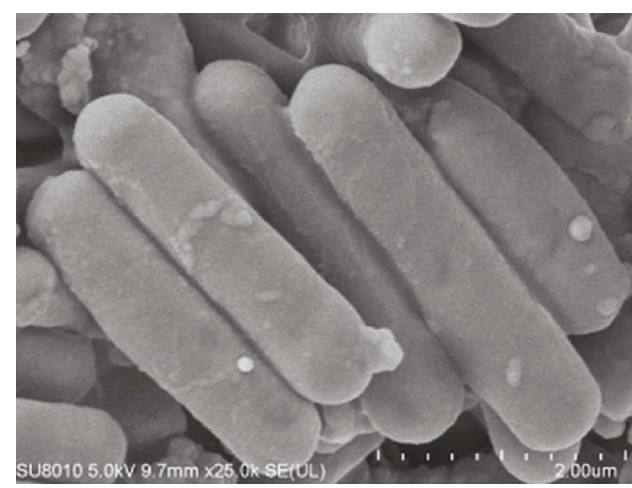

(a)

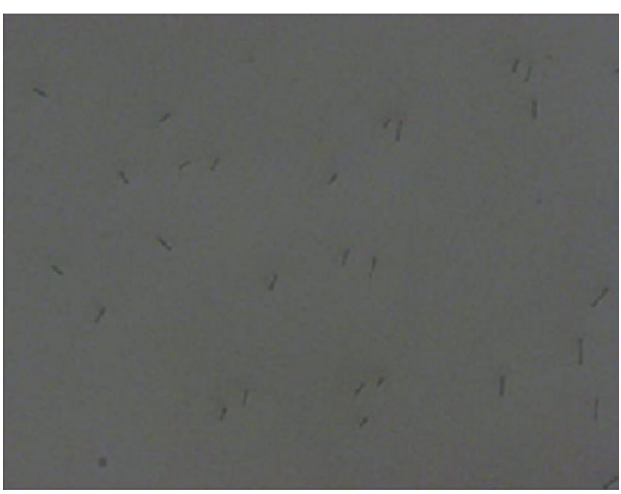

(c)

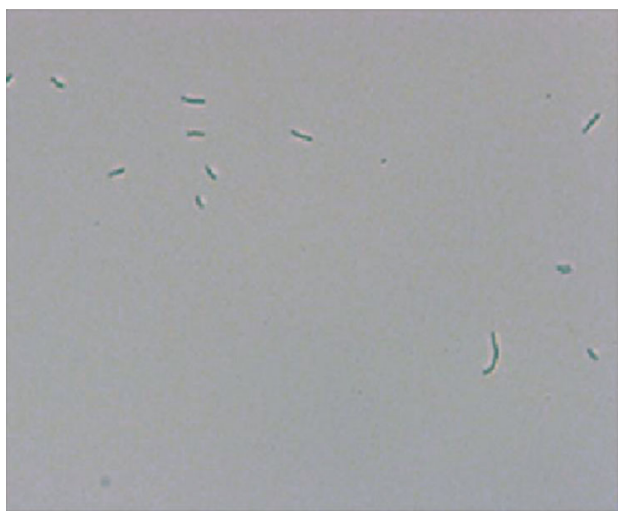

(e)

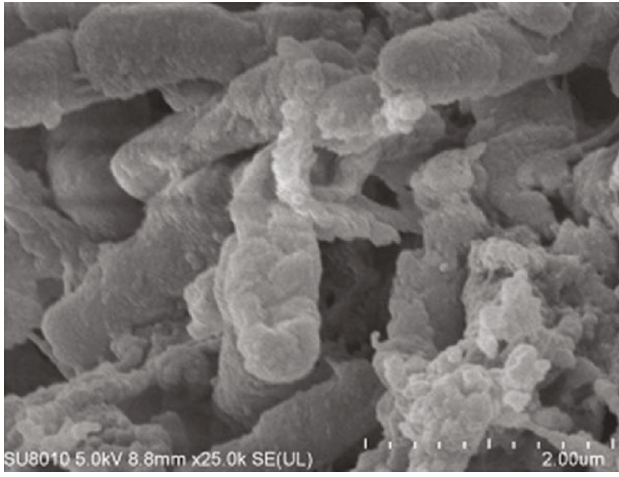

(b)

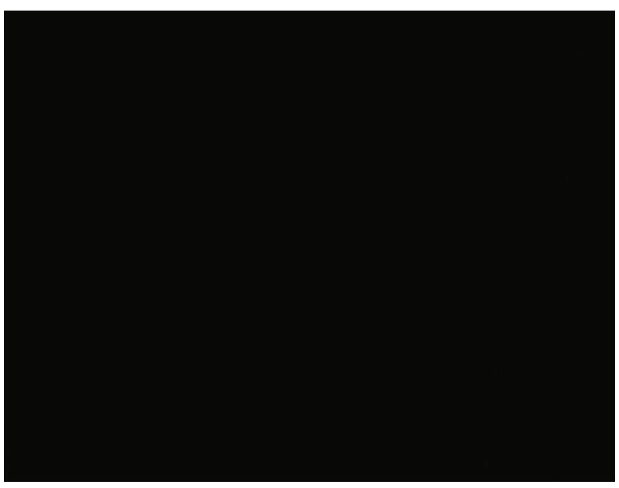

(d)

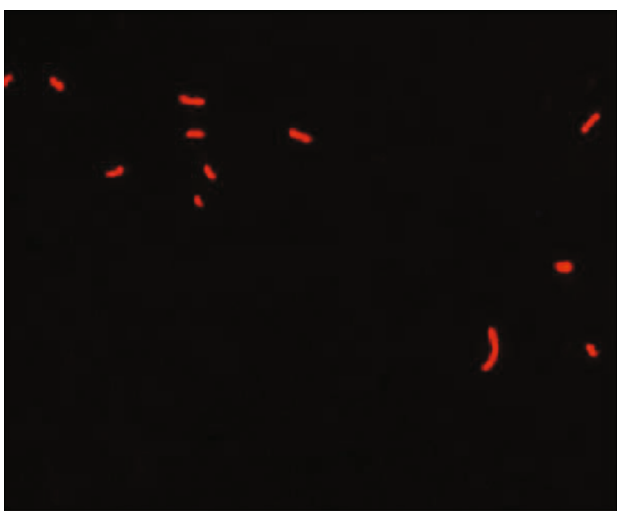

(f)

Figure 3: Morphological changes of $C$. difficile ATCC 9689 treated with 0.5 MIC of the C-1 lipopeptide. Observed with a scanning electron microscope $(\times 25000$; (a) untreated cells and (b) treated cells). Observed with a light microscope $(\times 400$; (c) untreated cells and (e) treated cells). Observed with a fluorescent microscope (PI staining, $\times 400$; (d) untreated cells and (f) treated cells).

TABLE 2: Genome project information summary of B. amyloliquefaciens C-1.

\begin{tabular}{lccc}
\hline Property/attributes & C-1 & Property/attributes & C-1 \\
\hline Finishing quality & High-quality draft & Total predicted CDS & 3805 \\
Sequencing platform & PacBio Sequel & rRNA operons & 27 \\
Total bases (Mb) & 757.4 & tRNA & 86 \\
NCBI taxonomy ID & 1386 & tmRNA & 1 \\
BioProject ID & PRJNA427474 & Noncoding RNA & 81 \\
Genome size (bp) & 3934216 & Miscellaneous RNA & 81 \\
GC content $(\%)$ & 46.5 & & \\
\hline
\end{tabular}




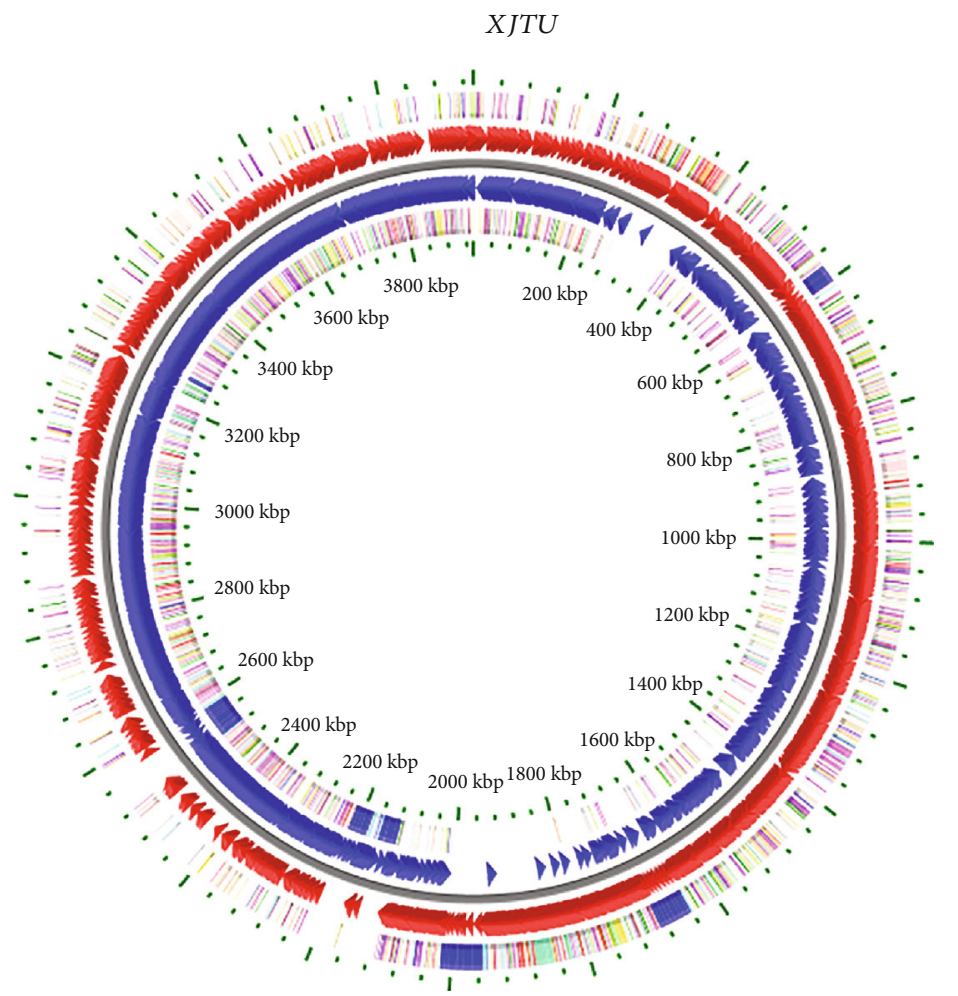

BASYS: Sunday March 05 01:31:32 2017

Length: 3,934,217 bp; Genes; 4,223

BASys

Cellularprocesses

Cell division and chromosome partitioning

Posttranslational modification, protein turnover, chaperones

Cell envelope biogenesis, outer membrane

Cell motility and secretion

Inorganic ion transport and metabolism

Signal transduction mechanisms

Metabolism

Energy production and conversion

- Carbohydrate transport and metabolism

- Amino acid transport and metabolism

Nucleotide transport and metabolism

Coenzyme metabolism

Lipid metabolism

Secondary metabolites biosynthesis, transport and catabolism
Genes encoding proteins

Fonvard strand

- Reverse strand

Genes encoding functional RNA

Fonvard strand

Reverse strand

COG functional categories

Information storage and processing

- Translation, ribosomal structure and biogenesis

- Transcription

DNA replication, recombination and repair

Poorly characterized

General function prediction only

Function unknown

(a)

Figure 4: Continued. 


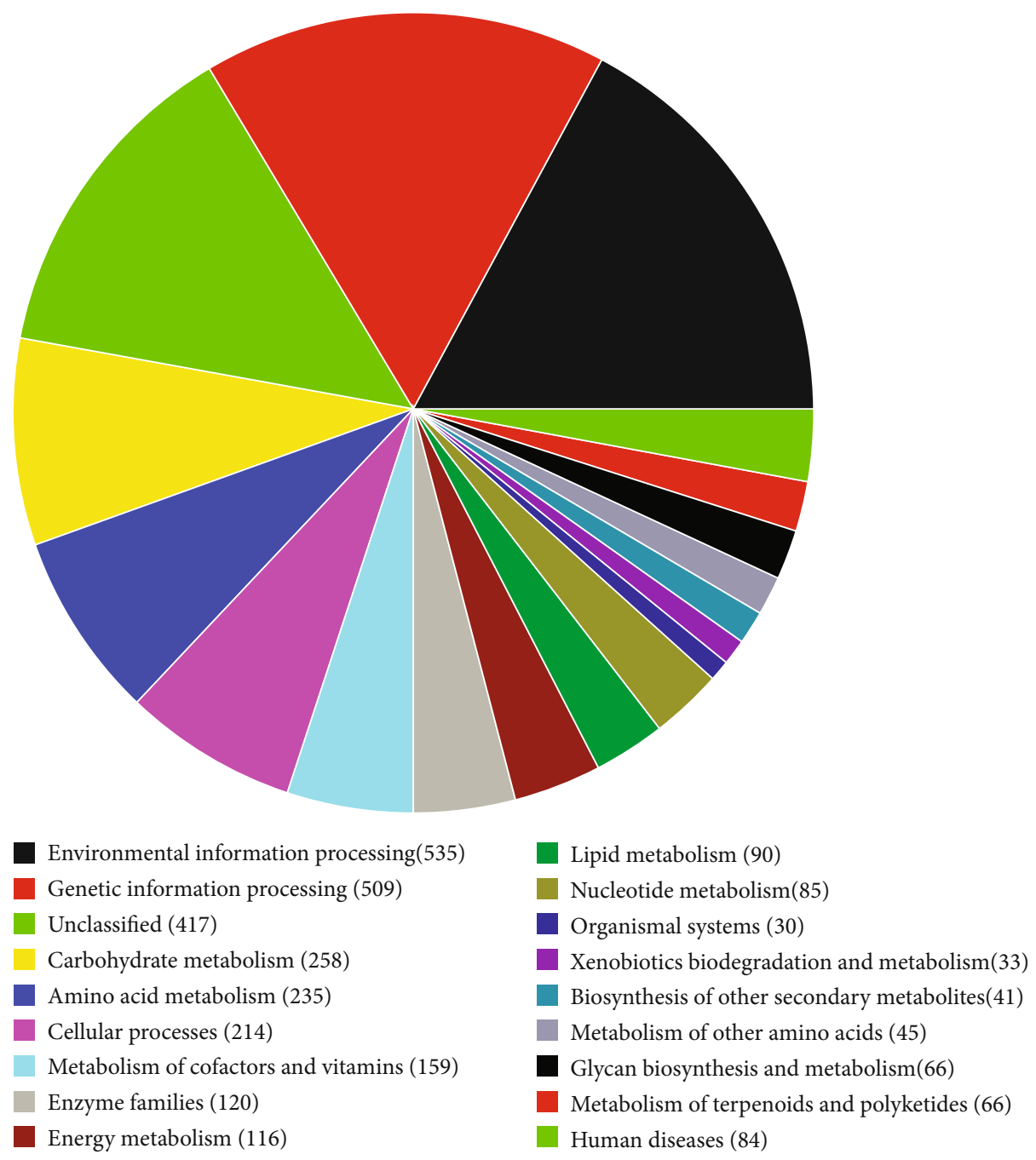

(b)

FIgURE 4: The genome map (a) of B. amyloliquefaciens C-1 and overview of the subsystem category coverage of the C-1 genome based on RAST serve (b). The red circle is the CDS of the forward strand, and the blue circle is the CDS of the reverse strand. The outer circle represents the categorization of predicted protein coding sequences in the C-1 genome in COG annotation, and the inner circle represents the genes encoding function RNA.

Blast searches of the $16 \mathrm{~S}$ rRNA gene sequence of C-1 showed that it was most similar to other B. amyloliquefaciens isolates. B. amyloliquefaciens isolates appear to group into two clades indicated by phylogenetic tree analysis (Fig S3). Although C-1 is in the same clade with known strains such as DSM7, ATCC19217, and ATCC 14580, it appears to be phylogenetically distant from most other isolates.

3.5. Secondary Metabolites from B. amyloliquefaciens Strains. As much as $8.5 \%$ of the $B$. amyloliquefaciens C-1 genome CDS was assigned to categories related to the secondary metabolites responsible for the control of pathogens ("Motility and Chemotaxis" [85 CDS], "Membrane Transport" [71 CDS], "Virulence, Disease and Defense" [70 CDS], "Secondary Metabolism" [6 CDS], and "Stress Responses" [108 CDS]). For the functional categories of genes, a possible role in bacteria inhibition may be important. In the carbohydrate transport and metabolism cate- gory, 437 genes (10.9\% of total genes) were predicted in the $\mathrm{C}-1$ genome. This finding suggests that $\mathrm{C}-1$ possesses a broad battery of genes coding for enzymes required to release a variety of environmental carbon sources.

As a Bacillus. spp., B. amyloliquefaciens possesses an enormous potential to synthesize bioactive secondary metabolites, especially nonribosomal-synthesized peptides and polyketides. For the nonribosomal peptide synthetases (NRPSs) and polyketide synthases (PKS), we used antiSMASH to identify related giant gene clusters (Table 3, Table S1). C-1 was found to harbor genes encoding six nonribosomal peptides (surfactins (srfABCD), iturins (ituABCD), fengycins (fenABCDE), bacillibactin (bmyABC), teichuronic, and bacilysin) and three polyketides (bacillaene (baeEDLMNJRS), difficidin (difABCDEFGHIJ), and macrolactin (mlnABCDEFGHI)). Compared with other sequenced B. amyloliquefaciens strains, fengycin, difficidin, bacillibactin, bacilysin, macrolactin, and bacillanen showed 
TABLE 3: Identification of gene clusters potentially involved in the synthesis of secondary metabolites by B. amyloliquefaciens C-1.

\begin{tabular}{|c|c|c|c|c|}
\hline Cluster $^{\mathrm{a}}$ & Type $^{\text {b }}$ & From $^{c}$ & $\mathrm{To}^{\mathrm{c}}$ & Secondary metabolite ${ }^{\mathrm{d}}$ \\
\hline 1 & Saccharide & 165858 & 190731 & Unknown \\
\hline 2 & NRPS & 556597 & 622004 & Surfactin \\
\hline 3 & Fatty acid & 785309 & 810347 & Unknown \\
\hline 4 & NRPS & 938299 & 967890 & Iturins \\
\hline 5 & Other KS & 1158436 & 1199680 & Butirosin \\
\hline 6 & Fatty acid & 1227747 & 1248724 & Unknown \\
\hline 7 & Terpene & 1281720 & 1302460 & Unknown \\
\hline 8 & Fatty acid & 1314519 & 1339344 & Citrulline \\
\hline 9 & Putative & 1378928 & 1396630 & Molybdenum cofactor \\
\hline 10 & Lantipeptide & 1406687 & 1451837 & Unknown \\
\hline 11 & Transatpks & 1624433 & 1706630 & Macrolactin \\
\hline 12 & Transatpks-NRPS & 1932737 & 2035409 & Bacillaene \\
\hline 13 & Transatpks-NRPS & 2100037 & 2237835 & Fengycin \\
\hline 14 & Terpene & 2263057 & 2284940 & Unknown \\
\hline 15 & T3PKS & 2348257 & 2389357 & Unknown \\
\hline 16 & Transatpks & 2504342 & 2604794 & Difficidin \\
\hline 17 & Bacteriocin-NRPS & 3235204 & 3301995 & Bacillibactin \\
\hline 18 & Saccharide & 3504667 & 3530078 & Unknown \\
\hline 19 & Saccharide & 3624018 & 3678829 & Teichuronic \\
\hline 20 & Saccharide & 3823278 & 3895655 & Bacilysin \\
\hline
\end{tabular}

${ }^{\mathrm{a}}$ Clusters identified using default settings of antiSMASH 5.0. ${ }^{\mathrm{b}}$ Class of gene cluster according to antiSMASH 5.0. ${ }^{\mathrm{c}}$ Location of gene clusters in the $B$. amyloliquefaciens $\mathrm{C}-1$ genome. ${ }^{\mathrm{d}}$ Secondary metabolites potentially produced based on the gene clusters.

$100 \%$ identity, whereas surfactins had $82 \%$ identity (Table S1). Surfactin can inhibit a wide range of microorganisms due to its ability to insert into the cell wall and create ion pores. Bacillomycin D, iturin, and fengycin have antifungal properties primarily based on their ability to disrupt the cell wall [30, 31]. Macrolactins, important 24-membered macrolactones produced by Bacillus spp., exhibit antimicrobial activities, where macrolactin $\mathrm{A}$ and $\mathrm{E}$ and succinyl macrolactin are the representative compounds. And it is assembled by a modular PKS system like macrolides, which could inhibit the $\mathrm{H}^{+}$-transporting ATPase of the bacterial cells [32]. Polyketide compounds inhibit a wide range of microorganisms by preventing protein synthesis [31]. The identity of surfactin among different strains varied from $82 \%$ to $96 \%$, including subunit genes of SrfAA, SrfAB, SrfAC, and SrfAD (Fig S4). C-1 had all the subunit genes, SrfAA, SrfAB, SrfAC, and SrfAD, and the mutation of subunit genes in other strains may indicate loss of the ability to synthesize secondary metabolites [33].

PCR experiments detected C-1 surfactin, iturin, and fengycin genes, an intact Bac operon that included Bacilysin biosynthesis proteins $\mathrm{BacA}, \mathrm{BacB}, \mathrm{BacC}, \mathrm{BacD}$, and $\mathrm{BacE}$ (ORF3909-3913), and the oligopeptide permease operon (ORF1362-1366). Bac proteins, nonribosomally synthesized dipeptides active against a range of bacteria and some fungi, are involved in the biosynthesis of bacilysin. The proteolysis of this dipeptide releases the nonproteinogenic amino acid L-anticapsin, which functions as a competitive inhibitor of glucosamine synthase and can cause lysis of fungal cells [34]. Because there was $100 \%$ sequence identity of bacilysin, whereas there was no antifungal activity of the C-1 lipopeptide and exopolysaccharide, the regulation and expression of the encoded Bac operon, and modification of produced bacilysin, deserve more analysis.

Other antimicrobial gene clusters were predicted by antiSMASH, such as the lantibiotic amylolysin [35], the bacteriocin amylocyclicin [36], and the aminoglycoside antibiotic butirosin [37]. These antibacterials have not been detected by chemical analysis from C- 1 supernatants, possibly because of the fermentation medium or condition that had been used [38]. Potential gene clusters may explain the broad activity of C-1 against pathogens.

We also detected in the C-1 genome other beneficial genes, such as phospholipase (ORF767) and siderophore production genes. There were seven gene clusters responsible for siderophore production and iron acquisition, including an ABC-type $\mathrm{Fe}^{3+}$-siderophore transport system (ORF1-2, ORF3417-3418, 4007-4008), an Fe-bacillibactin uptake system (ORF413-415), an iron compound ABC uptake transporter (ORF624-627), and a siderophore biosynthesis protein (ORF1221-1222, 3262-3267). These proteins enable bacteria to sequester iron complexes produced by other pathogens and antagonize certain pathogens [38, 39].

Moreover, we also detected genes for amphiphilic membrane-active biosurfactants and peptide antibiotics that have powerful antibacterial and mosquito larvicidal activity. The giant gene clusters add to the capacity of the $\mathrm{C}-1$ bacterium to contribute to the antimicrobial activity against C. difficile. And we also checked the biosafety of the C-1 strain by using Galleria mellonella and intestinal epithelial 
cells, which all indicated the safety of B. amyloliquefaciens C-1. A future study of how these gene clusters are expressed and regulated will help explain the synthesis of antimicrobial lipopeptides and augment our knowledge for the control of CDI.

\section{Conclusions}

B. amyloliquefaciens $\mathrm{C}-1$ fermentation supernatant contains a mixture of lipopeptides, namely, surfactin and fengycin, which had a strong inhibitory effect on $C$. difficile growth and viability. Systematic research of the antibacterial mechanism showed that the $\mathrm{C}-1$ lipopeptide damages the integrity and permeability barrier of the cell wall and cell membrane, then leads to $C$. difficile cell death. The $\sim 3.93 \mathrm{Mbp}$ genome of $\mathrm{C}-1$ reveals the genetic basis of its antimicrobial activity, and the antimicrobial compound-encoding gene clusters provide better understanding of the antibacterial mechanisms of this strain. Furthermore, the genome analysis will facilitate the production of effective probiotics that inhibit multidrug resistant pathogens in the host intestinal ecosystem, especially the phospholipase- and siderophoreproducing clusters. Until now, the anti-C. difficile activities of the bacteriocins were known predominantly from in vitro studies; thus, the in vivo efficacies of the majority of these bacteriocins deserve further investigation.

\section{Data Availability}

The data used to support the findings of this study are available from the corresponding author upon request.

\section{Conflicts of Interest}

The authors declare no conflict of interest.

\section{Authors' Contributions}

Jia Lv and Rong Da contributed equally to this work.

\section{Acknowledgments}

This work was financially supported by the National Natural Science Foundation of China (81673199) and the National Science Basic Research Plan in Shaanxi Province of China (2018JM7054).

\section{Supplementary Materials}

Fig S1: the isolation, purification, and verification of the $B$. amyloliquefaciens $\mathrm{C}-1$ lipopeptide. (A) The acid precipitated crude lipopeptide; (B) the UV-VIS spectrophotometer scanning analysis of crude lipopeptide; (C) the purified lipopeptide isolated by a TLC plate; (D) the PCR detection of lipopeptide synthesis-related genes fenB, srf, and ituD in the C-1 genome; phylogenetic tree of PCR fragments fen $\mathrm{B}$ (E), srf (F), and ituD (G). Fig S2: effect of the $\mathrm{C}-1$ lipopeptide on AKPase in C. difficile ATCC $9689\left({ }^{*} P<0.05,{ }^{* *} P<0.01\right.$ indicated statistically significant differences of C-1 lipopeptide treatments vs. negative control). Fig S3: neighbor- joining phylogenetic tree based on $16 \mathrm{~S}$ rRNA gene sequences of B. amyloliquefaciens strains. 16S rRNA gene sequences were from 16S ribosomal RNA gene partial sequence or directed from the genome annotation in NCBI with accession numbers of C-1 (JX028840), LL3 (CP002634.1), XH7 (CP002927.1), ATCC 13952 (CP009748.1), DSM7 (NC 014551), SRCM101267 (CP021505.1), Y2 (HE774679.1), UMAF6614 (NZ_CP006960), 19217 (CP009749.1), TA208 (CP002627.1), ATCC 14580 (CP000002.3), RD7-7 (CP016913.1), S499 (CP014700.1), LFB112 (NC_023073), CC178 (CP006845.1), L-H15 (CP010556.1), L-S60 (CP011278.1), B15 (KT923051.1), KHG19 (NZ_CP007242), ATCC 7050 (DQ297928.1), Y14 (NZ_CP017953), 168 (NC_ 000964), IT45 (NC_020272), ATCC 14581 (JQ579621.1), WS-8 (CP018200.1), LM2303 (MN640968.1), UMAF6639 (GCA_001593765), and DSM 319 (KM051080.1). Fig S4: phylogenetic tree of genome-sequenced B. amyloliquefaciens strains based on the amino acid sequences of surfactin synthetases SrfAA, SrfAB, SrfAC, and SrfAD, and the comparison of the gene loci strain C-1 (SRP127533), KHG19 (NZ CP007242), UMAF6639 (GCA_001593765), UMAF6614 (NZ_CP006960), LFB112 (NC_023073), IT45 (NC_ 020272), DSM7 (NC_014551), Y14 (NZ_CP017953), and 168 (NC_000964). Table S1: comparison of gene clusters potentially involved in the synthesis of secondary metabolites in B. amyloliquefaciens-sequenced strains. (Supplementary Materials)

\section{References}

[1] Z. Peng, L. Ling, C. W. Stratton et al., "Advances in the diagnosis and treatment ofClostridium difficileinfections," Emerging Microbes \& Infections, vol. 7, no. 1, pp. 1-13, 2018.

[2] A. C. Clements, R. J. Magalhães, A. J. Tatem, D. L. Paterson, and T. V. Riley, "Clostridium difficile PCR ribotype 027: assessing the risks of further worldwide spread," Lancet Infectious Diseases, vol. 10, no. 6, pp. 395-404, 2010.

[3] D. W. Hecht, M. A. Galang, S. P. Sambol, J. R. Osmolski, S. Johnson, and D. N. Gerding, "In vitro activities of 15 antimicrobial agents against 110 toxigenic Clostridium difficile clinical isolates collected from 1983 to 2004," Antimicrobial Agents and Chemotherapy, vol. 51, no. 8, pp. 2716-2719, 2007.

[4] C. F. Manthey, L. Eckmann, and V. Fuhrmann, "Therapy forClostridium difficileinfection - any news beyond metronidazole and vancomycin?," Expert Review of Clinical Pharmacology, vol. 10, no. 11, pp. 1239-1250, 2017.

[5] N. Roshan, K. A. Hammer, and T. V. Riley, "Non-conventional antimicrobial and alternative therapies for the treatment of Clostridium difficile infection," Anaerobe, vol. 49, pp. 103$111,2018$.

[6] S. Torsten, "Bacillus subtilis antibiotics: structures, syntheses and specific functions," Molecular Microbiology, vol. 56, no. 4, pp. 845-857, 2005.

[7] I. Dimkić, S. Stanković, M. Nišavić et al., "The profile and antimicrobial activity of Bacillus lipopeptide extracts of five potential biocontrol strains," Frontiers in Microbiology, vol. 8, 2017.

[8] B. C. S. Farias, D. C. Hissa, C. T. M. do Nascimento et al., "Cyclic lipopeptide signature as fingerprinting for the screening of halotolerant Bacillus strains towards microbial 
enhanced oil recovery," Applied Microbiology and Biotechnology, vol. 102, no. 3, pp. 1179-1190, 2018.

[9] D. B. Abdallah, O. Frikha-Gargouri, and S. Tounsi, "Bacillus amyloliquefaciens strain 32a as a source of lipopeptides for biocontrol of Agrobacterium tumefaciens strains," Journal of Applied Microbiology, vol. 119, no. 1, pp. 196-207, 2015.

[10] S. Geeraerts, R. Ducatelle, F. Haesebrouck, and F. Van Immerseel, "Bacillus amyloliquefaciens as prophylactic treatment for Clostridium difficile-associated disease in a mouse model," Journal of Gastroenterology and Hepatology, vol. 30, no. 8, pp. 1275-1280, 2015.

[11] J. Lv, X. K. Xu, S. K. Hu, R. J. Zhang, and B. Han, "Isolation and identification of antibacterial lipopeptide produced by Bacillus amyloliquefaciens C-1," Natural Product Research and Development, vol. 27, no. 2, pp. 199-204, 2015.

[12] W. J. Zhou, J. Yang, Y. Wan, R. J. Zhang, and B. Han, “Antimicrobial activity on foodborne pathogens of extracellular fermented products from Bacillus amyloliquefaciens C-1," Natural Product Research and Development, vol. 26, no. 1, pp. 123-127, 2014.

[13] L. Belbahri, A. Chenari Bouket, I. Rekik et al., "Comparative genomics of Bacillus amyloliquefaciens strains reveals a core genome with traits for habitat adaptation and a secondary metabolites rich accessory genome," Frontiers in Microbiology, vol. 8, no. 8, 2017.

[14] X. Zhang, B. Li, Y. Wang et al., "Lipopeptides, a novel protein, and volatile compounds contribute to the antifungal activity of the biocontrol agent Bacillus atrophaeus CAB-1," Applied Microbiology and Biotechnology, vol. 97, no. 21, pp. 95259534, 2013.

[15] Y. Han, B. Zhang, Q. Shen et al., "Purification and identification of two antifungal cyclic peptides produced by Bacillus amyloliquefaciens L-H15," Applied Biochemistry and Biotechnology, vol. 176, no. 8, pp. 2202-2212, 2015.

[16] I. Wiegand, K. Hilpert, and R. E. Hancock, "Agar and broth dilution methods to determine the minimal inhibitory concentration (MIC) of antimicrobial substances," Nature Protocols, vol. 3, no. 2, pp. 163-175, 2008.

[17] J. S. Eom and H. S. Choi, "Inhibition of Bacillus cereus growth and toxin production by Bacillus amyloliquefaciens RD7-7 in fermented soybean products," Journal of Microbiology and Biotechnology, vol. 26, no. 1, pp. 44-55, 2016.

[18] D. R. Snydman, N. V. Jacobus, and L. A. McDermott, "Activity of a novel cyclic lipopeptide, CB-183,315, against resistant Clostridium difficile and other Gram-positive aerobic and anaerobic intestinal pathogens," Antimicrobial Agents and Chemotherapy, vol. 56, no. 6, pp. 3448-3452, 2012.

[19] H. M. Xu, Y. J. Rong, M. X. Zhao, B. Song, and Z. M. Chi, "Antibacterial activity of the lipopeptides produced by Bacillus amyloliquefaciens M1 against multidrug-resistant Vibrio spp. isolated from diseased marine animals," Applied Microbiology and Biotechnology, vol. 98, no. 1, pp. 127-136, 2014.

[20] C. Ma, N. He, Y. Zhao, D. Xia, J. Wei, and W. Kang, "Antimicrobial mechanism of hydroquinone," Applied Biochemistry and Biotechnology, vol. 189, no. 4, pp. 1291-1303, 2019.

[21] B. Zhang, C. Dong, Q. Shang, Y. Han, and P. Li, "New insights into membrane-active action in plasma membrane of fungal hyphae by the lipopeptide antibiotic bacillomycin L," Biochimica et Biophysica Acta, vol. 1828, no. 9, pp. 2230-2237, 2013.
[22] R. D. Finn, J. Clements, and S. R. Eddy, "HMMER web server: interactive sequence similarity searching," Nucleic Acids Research, vol. 39, suppl, pp. W29-W37, 2011.

[23] C. Camacho, G. Coulouris, V. Avagyan et al., "BLAST+: architecture and applications," BMC Bioinformatics, vol. 10, no. 1, p. 421, 2009.

[24] K. Blin, M. H. Medema, R. Kottmann, S. Y. Lee, and T. Weber, "The antiSMASH database, a comprehensive database of microbial secondary metabolite biosynthetic gene clusters," Nucleic Acids Research, vol. 45, no. D1, pp. D555-D559, 2017.

[25] J. Cheetham, F. Dehne, S. Pitre, A. Rau-Chaplin, and P. J. Taillon, "Parallel CLUSTAL W for PC Clusters," in Computational Science and Its Applications - ICCSA 2003, pp. 300-309, Springer, Berlin, Heidelberg, 2003.

[26] K. Tamura, D. Peterson, N. Peterson, G. Stecher, M. Nei, and S. Kumar, "MEGA5: molecular evolutionary genetics analysis using maximum likelihood, evolutionary distance, and maximum parsimony methods," Molecular Biology and Evolution, vol. 28, no. 10, pp. 2731-2739, 2011.

[27] P. Jin, H. Wang, W. Liu, and W. Miao, "Characterization of lpaH2 gene corresponding to lipopeptide synthesis in Bacillus amyloliquefaciens HAB-2," BMC Microbiology, vol. 17, no. 1, p. 227, 2017.

[28] X. Li, C. He, L. Song et al., "Antimicrobial activity and mechanism of Larch bark procyanidins against Staphylococcus aureus," Acta Biochimica et Biophysica Sinica, vol. 49, no. 12, pp. 1058-1066, 2017.

[29] A. Etchegaray, C. de Castro Bueno, I. S. de Melo et al., "Effect of a highly concentrated lipopeptide extract of Bacillus subtilis on fungal and bacterial cells," Archives of Microbiology, vol. 190, no. 6, pp. 611-622, 2008.

[30] B. J. Schofield, A. Skarshewski, N. Lachner et al., "Near complete genome sequence of the animal feed probiotic, Bacillus amyloliquefaciens H57," Standards in Genomic Sciences, vol. 11, no. 1, p. 60, 2016.

[31] D. H. Kim, H. K. Kim, K. M. Kim et al., "Antibacterial activities of macrolactin A and 7-O-succinyl macrolactin A from Bacillus polyfermenticus KJS-2 against vancomycin-resistant enterococci and methicillin-resistant Staphylococcus aureus," Archives of Pharmacal Research, vol. 34, no. 1, pp. 147-152, 2011.

[32] X. Yan, Y.-X. Zhou, X.-X. Tang et al., "Macrolactins from marine-derived Bacillus subtilis B5 bacteria as inhibitors of inducible nitric oxide and cytokines expression," Marine Drugs, vol. 14, no. 11, p. 195, 2016.

[33] B. Fan, J. Blom, H. P. Klenk, and R. Borriss, "Bacillus amyloliquefaciens, Bacillus velezensis, and Bacillus siamensis form an "Operational Group B. amyloliquefaciens" within the B. subtilis species complex," Frontiers in Microbiology, vol. 8, 2017.

[34] I. Martínez-Raudales, Y. de la Cruz-Rodríguez, A. AlvaradoGutiérrez et al., "Draft genome sequence of Bacillus velezensis 2A-2B strain: a rhizospheric inhabitant of Sporobolus airoides (Torr.) Torr., with antifungal activity against root rot causing phytopathogens," Standards in Genomic Sciences, vol. 12, no. $1,2017$.

[35] A. A. Arias, M. Ongena, B. Devreese, M. Terrak, B. Joris, and P. Fickers, "Characterization of amylolysin, a novel lantibiotic from Bacillus amyloliquefaciens GA1," PLoS One, vol. 8, no. 12, 2013. 
[36] R. Scholz, J. Vater, A. Budiharjo et al., "Amylocyclicin, a novel circular bacteriocin produced by Bacillus amyloliquefaciens FZB42," Journal of Bacteriology, vol. 196, no. 10, pp. 184211852, 2014.

[37] N. M. Llewellyn, Y. Li, and J. B. Spencer, "Biosynthesis of butirosin: transfer and deprotection of the unique amino acid side chain," Chemistry \& Biology, vol. 14, no. 4, pp. 379-386, 2007.

[38] S. B. Khedher, H. Boukedi, O. Kilani-Feki et al., "Bacillus amyloliquefaciens AG1 biosurfactant: Putative receptor diversity and histopathological effects on Tuta absoluta midgut," Journal of Invertebrate Pathology, vol. 132, pp. 42-47, 2015.

[39] D. Wang, Y. Zhan, D. Cai, X. Li, Q. Wang, and S. Chen, "Regulation of the synthesis and secretion of the iron chelator cyclodipeptide pulcherriminic acid in Bacillus licheniformis," Applied and Environmental Microbiology, vol. 84, no. 13, 2018. 